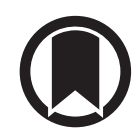

CrossMark

\section{Predictors of mortality for patients with COVID-19 pneumonia caused by SARS-CoV-2}

\author{
To the Editor:
}

As an emerging infectious disease, coronavirus disease 2019 (COVID-19) pneumonia, which is caused by the novel severe acute respiratory syndrome coronavirus 2 (SARS-CoV-2) has resulted in a severe global public health emergency. According to the World Health Organization (WHO) COVID-19 epidemic interactive dashboard, as of 19 June 2020, there have been 8385440 confirmed cases all over the world, including 450686 deaths. Under such urgent conditions, it is of great clinical significance to distinguish patients with poor clinical outcome (such as severe, critical or death) from within the large number of patients with COVID-19 using regular parameters (such as demographic data, past health history, and common laboratory examination results). Du et al. [1] performed a single centre prospective cohort study to investigate the possible risk factors associated with the poorest clinical outcome (dying from COVID-19 pneumonia). They reported that age $\geqslant 65$ years, pre-existing concurrent cardiovascular or cerebrovascular diseases, $\mathrm{CD} 3+\mathrm{CD} 8+\mathrm{T}$-cells $\leqslant 75$ cells $\mu \mathrm{L}^{-1}$ and cardiac troponin $\mathrm{I} \geqslant 0.05 \mathrm{ng} \cdot \mathrm{mL}^{-1}$ in patients with COVID-19 pneumonia were associated with increased risk of death from this disease [1]. They further identified that CD3+CD8+ T-cells $\leqslant 75$ cells $\mu \mathrm{L}^{-1}$ and cardiac troponin I especially $\geqslant 0.05 \mathrm{ng} \cdot \mathrm{mL}^{-1}$ could be used as predictors for mortality of patients with COVID-19 pneumonia using a matched case-control study [1]. With great interest, we have read the full text of the paper and found that there are several issues which are worth clarifying.

First, both in the Abstract and Results sections, Du et al. [1] reported that pre-existing cardiovascular or cerebrovascular diseases in patients with COVID-19 pneumonia was associated with elevated risk of dying from this disease using multivariate logistic regression analysis, with odds ratio and 95\% confidence interval being equal to $2.464(0.755-8.044)$ and $p$-value being equal to 0.007 . Obviously, the $95 \%$ confidence interval includes 1 , indicating that the estimated population odds ratio is possible to be equal to 1. According to the principle of statistics, the conclusion made from parameter estimation and hypothesis test should be consistent. In other words, if the p-value for odds ratio of pre-existing cardiovascular or cerebrovascular disease is 0.007 , the lower limit of the $95 \%$ confidence interval should at least be more than 1, considering this factor is risk one for poor clinical outcome. Thus, we suggest that the authors should check the data and to ascertain whether there were typography errors or an incorrectly interpreted statistical result.

Second, in the Methods section, Du et al. [1] state "The information for all patients, including demographic data, clinical characteristics, laboratory parameters and outcomes, were collected prospectively." And in the Results section, the authors showed the summary statistics for demographic data, life sign data and laboratory examination data in tables 1 and 2 of their original article [1]. However, the authors did not denote definitively, in the summary statistics for laboratory findings and life sign data, on which time of the patients' examination the results were based. In clinical practice, it is common that one patient with COVID-19 pneumonia receives laboratory examinations (such as blood regular test) several times, and the results of the same test for the same patient may be different from one time to another.

@ERSpublications

There are several issues which are worth clarifying in the paper "Predictors of mortality for patients with COVID-19 pneumonia caused by SARS-CoV-2: a prospective cohort study" published in the European Respiratory Journal https://bit.ly/336rv2Y

Cite this article as: Yang $\mathrm{H}-\mathrm{J}$, Zhang $\mathrm{Y}-\mathrm{M}$, Yang $\mathrm{M}$, et al. Predictors of mortality for patients with COVID-19 pneumonia caused by SARS-CoV-2. Eur Respir J 2020; 56: 2002439 [https://doi.org/ 10.1183/13993003.02439-2020]. 
Third, in the Discussion section, the authors state "As of midnight on 24 March 2020, the numbers of Chinese confirmed COVID-19 pneumonia cases and deaths were 81218 and 3281, respectively, indicating that the overall death rate from COVID-19 pneumonia was $4 \%$." Obviously, the so-called overall death rate of $4 \%$ was calculated using the formula: number of cumulative deaths/cumulative number of confirmed cases, i.e. 3281/81218. This simple formula is problematic, especially considering the COVID-19 epidemic was ongoing then. As such there was still a certain proportion of COVID-19 patients (confirmed on or before 24 March 2020) whose clinical outcomes were unknown. As the authors said in the latter part of their Discussion section, "On 24 March 2020, China had 4287 current cases with confirmed COVID-19 pneumonia, and 1399 (32.6\%) of them were very severe cases." We think that using case fatality rate (CFR) to replace death rate or mortality rate in the Discussion section of the report by Du et al. [1] is appropriate. Death rate or mortality rate of a certain disease is often defined as the ratio of deaths from a certain disease in an area to the population of that area. CFR refers to the proportion of cases who eventually die from the disease. To estimate the CFR of COVID-19 pneumonia, Du et al. [1] can refer to the methods proposed by GHANi et al. [2].

Taken together, the findings of the study reported by Du et al. [1] are of great significance, though some possible error and inappropriate expression were found. We hope our comments will be helpful to improve the expression and increase the quality of the published paper.

Hai-Jun Yang $\oplus^{1,2}$, Yan-Mei Zhang ${ }^{1}$, Min Yang $\oplus^{1,2}$ and Xing Huang ${ }^{1}$

${ }^{1}$ Dept of Preventive Medicine, College of Basic Medicines, Hubei University of Chinese Medicine, Wuhan, China. ${ }^{2}$ Institute of Epidemic Research, Hubei University of Chinese Medicine, Wuhan, China.

Correspondence: Hai-Jun Yang, Dept of Preventive Medicine, College of Basic Medicines, Hubei University of Chinese Medicine, No. 16 Huangjiahu West Road, Hongshan District, Wuhan 430065, China. E-mail: haijyang@126.com

Received: 22 June 2020 | Accepted: 15 July 2020

Conflict of interest: None declared.

\section{References}

1 Du RH, Liang LR, Yang CQ, et al. Predictors of mortality for patients with COVID-19 pneumonia caused by SARS-CoV-2: a prospective cohort study. Eur Respir J 2020; 55: 2000524.

2 Ghani AC, Donnelly CA, Cox DR, et al. Methods for estimating the case fatality ratio for a novel, emerging infectious disease. Am J Epidemiol 2005; 162: 479-486.

Copyright CERS 2020. This version is distributed under the terms of the Creative Commons Attribution Non-Commercial Licence 4.0.

From the authors:

We appreciate the thoughtful comments of H-J. Yang and co-workers in their correspondence addressing the predictors of mortality for patients with coronavirus disease 2019 (COVID-19) pneumonia caused by severe acute respiratory syndrome coronavirus 2 (SARS-CoV-2). Their comments are very helpful to improve the expression and increase the quality of our paper [1].

We agree that the first question of the 95\% confidence interval of pre-existing cardiovascular or cerebrovascular diseases was incorrect. Since all indicators are in the multivariate logistic regression analysis, the $95 \%$ confidence intervals of the other three indicators are modified. We have corrected the $95 \%$ confidence intervals for age $\geqslant 65$ years, pre-existing concurrent cardiovascular or cerebrovascular diseases, $\mathrm{CD}^{+} \mathrm{CD}^{+} \mathrm{T}$-cells $\leqslant 75$ cells $\cdot \mu \mathrm{L}^{-1}$ and cardiac troponin $\mathrm{I} \geqslant 0.05 \mathrm{ng} \cdot \mathrm{mL}^{-1}$ in a revised version of the table according to the the author correction notice in this issue of the European Respiratory Journal. Many studies have found the same results, that prevalent cardiovascular disease is associated with higher mortality and severity of COVID-19 [2-4]. Furthermore, SHI et al. [5] found that patients with cardiac injury (elevated TnI) have a worse prognosis, suggesting specific target organ damage by SARS-CoV-2. Our findings were likely to be generalisable to other populations worldwide.

@ERSpublications

Author response to the correspondence regarding "Predictors of mortality for patients with COVID-19 pneumonia caused by SARS-CoV-2: a prospective cohort study" https://bit.ly/31X8cal

Cite this article as: $\mathrm{Du} \mathrm{R}-\mathrm{H}$, Liang L-R, Yang C-Q, et al. Predictors of mortality for patients with COVID-19 pneumonia caused by SARS-CoV-2. Eur Respir J 2020; 56: 2002961 [https://doi.org/ 10.1183/13993003.02961-2020]. 
We completely agree with H-J. Yang and co-workers that in clinical practice, a patient may undergo some laboratory examinations (such as blood regular test) several times, and the results of the same test for the same patient may be different from one time to another. The information for all patients, including demographic data, clinical characteristics, laboratory parameters and outcomes, were collected prospectively upon hospital admission in our study. We further add the time of that laboratory parameters were obtained in the methods.

Overall death rate is the ratio of the number of dead individuals in a certain period to the average population number in the same period. The main difference between overall death rate and case fatality rate $(\mathrm{CRF})$ is that overall death rate refers to the frequency of death from a certain disease at a certain time, while CRF is used to describe the severity of a certain disease. We have carefully studied the methods for estimating the case fatality ratio for a novel, emerging infectious disease [6]. As of midnight on 24 March 2020, the numbers of Chinese confirmed COVID-19 pneumonia cases were 81218 and deaths were 3281. The confirmed case fatality rate was calculated using the formula: number of cumulative death/ cumulative number of confirmed cases. We consent that the overall death rate from COVID-19 pneumonia is an incorrect expression. So, the overall death rate has been corrected to "confirmed case fatality rate".

Rong-Hui Du ${ }^{1,3}$, Li-Rong Liang ${ }^{2,3}$, Cheng-Qing Yang ${ }^{1,3}$, Wen Wang ${ }^{2,3}$, Tan-Ze Cao ${ }^{1}$, Ming $\mathrm{Li}^{1}$, Guang-Yun Guo ${ }^{1}$, Juan Du ${ }^{1}$, Chun-Lan Zheng ${ }^{1}$, Qi Zhu ${ }^{1}$, Ming Hu ${ }^{1}$, Xu-Yan $\mathrm{Li}^{2}$, Peng Peng ${ }^{1,4}$ and Huan-Zhong Shi $\circledast^{2,4}$

${ }^{1}$ Dept of Respiratory and Critical Care Medicine, Wuhan Pulmonary Hospital, Wuhan, China. ${ }^{2}$ Dept of Respiratory and Critical Care Medicine, Beijing Chao-Yang Hospital, Capital Medical University, Beijing, China. ${ }^{3}$ These authors contributed equally. ${ }^{4}$ These authors are joint principal authors.

Correspondence: Huan-Zhong Shi, Dept of Respiratory and Critical Care Medicine, Beijing Chao-Yang Hospital, Capital Medical University, 8 Gongti Nanlu, Chao-Yang District, Beijing 100020, China. E-mail: shihuanzhong@sina.com

Received: 29 July 2020 | Accepted after revision: 10 Aug 2020

Conflict of interest: None declared.

\section{References}

1 Du RH, Liang LR, Yang CQ, et al. Predictors of mortality for patients with COVID-19 pneumonia caused by SARS-CoV-2: a prospective cohort study. Eur Respir J 2020; 55: 2000524.

$2 \mathrm{Wu} \mathrm{Z}$, McGoogan JM. Characteristics of and important lessons from the coronavirus disease 2019 (COVID-19) outbreak in China: summary of a report of 72314 cases from the Chinese Center for Disease Control and Prevention. JAMA 2020; 13: 1239-1242.

3 Ruan Q, Yang K, Wang W, et al. Clinical predictors of mortality due to COVID-19 based on an analysis of data of 50 patients from Wuhan, China. Intensive Care Med 2020; 46: 846-848.

4 Yang J, Zheng Y, Gou X, et al. Prevalence of comorbidities and its effects in coronavirus disease 2019 patients: a systematic review and meta-analysis. Int J Infect Dis 2020; 94: 91-95.

5 Shi S, Qin M, Shen B, et al. Association of cardiac injury with mortality in hospitalized patients with COVID-19 in Wuhan, China. JAMA Cardiol 2020; 5: 802-810.

6 Ghani AC, Donnelly CA, Cox DR, et al. Methods for estimating the case fatality ratio for a novel, emerging infectious disease. Am J Epidemiol 2005; 162: 479-486. 